\title{
A Real-Time Estimation Method of Roll Angle and Angular Rate Based on Geomagnetic Information
}

\author{
Lizhen Gao, ${ }^{1,2}$ Yingying Zhang $\mathbb{D}^{1},{ }^{1}$ Xiaoming Zhang, ${ }^{1,2}$ and Yuyang Xue ${ }^{1}$ \\ ${ }^{1}$ National Key Laboratory for Electronic Measurement Technology, North University of China, Taiyuan 030051, China \\ ${ }^{2}$ Key Laboratory of Instrumentation Science \& Dynamic Measurement of Ministry of Education, North University of China, \\ Taiyuan 030051, China \\ Correspondence should be addressed to Yingying Zhang; 19834406826@163.com
}

Received 22 August 2020; Revised 6 October 2020; Accepted 13 October 2020; Published 27 October 2020

Academic Editor: Tao Zhang

Copyright (C) 2020 Lizhen Gao et al. This is an open access article distributed under the Creative Commons Attribution License, which permits unrestricted use, distribution, and reproduction in any medium, provided the original work is properly cited.

\begin{abstract}
In the course of the guidance transformation of the rotating projectile, the accurate acquisition of the roll angle and roll angle rate is very important to the attitude determination and guidance control of the rotating projectile. However, due to the impact of high rotation and high overload of projectile, MEMS gyros have problems such as limited range, saturation, overload, and even performance degradation, which make the roll angle rate unable to be output normally. At the same time, because the MEMS gyro estimation of roll angle is in the form of angular rate integral, the roll angle cannot be estimated normally if the roll angle rate cannot be accurately obtained. In order to solve this problem, a real-time estimation of projectile roll angle and roll rate based on geomagnetic information under high dynamic and high overload conditions is presented. Firstly, according to the motion characteristics of the rotating projectile, the motion model of the projectile is established, and the roll angle and roll angle rate of the projectile are estimated by Kalman filtering algorithm under the conditions of high axial rotation and high overload. Considering the high dynamic characteristics of the rotating projectile, based on the Kalman filter, the algorithm of the forgetting filter with the forgetting factor is further adopted to estimate the roll angle and roll angle rate, so as to reduce the error caused by the estimation delay in the process of high-speed dynamic change. Simulation data and semiphysical test results show that the accuracy of roll angle estimated by this method reaches about $2^{\circ}$ in semiphysical test, which is one time higher than that calculated by the system. In the semiphysical experiment, the accuracy of the estimated roll rotation rate reaches $5 \%$, which is more than 6 times higher than that obtained by direct derivation. In the high dynamic stage, compared with the pure Kalman filter, the accuracy of roll angle with forgetting factor estimation is improved by an order of magnitude, and the accuracy of roll angle rate is improved by 4 times, which meets the desired accuracy of rotating projectile.
\end{abstract}

\section{Introduction}

In the course of the guidance transformation of conventional ammunition, there are some problems such as high rotational speed and high dynamic state in the flight state of the projectile so that the gyro cannot accurately measure the angular velocity of the projectile body roll. For example, in the case of a rotating projectile, the maximum axial rotation speed of the projectile can be up to $200 \mathrm{r} / \mathrm{s}$ during the launching process, and it is accompanied by a large change. In this case of high dynamic and high rotation speed, the ordinary MEMS gyro is already saturated and cannot normally measure angular rate and solve the roll angle. The acquisition of the roll angle of the projectile is the key technology of the control of the rotating projectile channel. Only by acquiring the roll angle of the projectile accurately can the angle position of the steering gear be determined, so as to guide the projectile and hit the target accurately through the control system of the guided ammunition. At the same time, the acquisition of the roll angular rate is to provide damping loop for the projectile control system and further improve the dynamic performance of projectile control, which is one of the essential factors in the process of projectile control. Therefore, it is necessary to have a method to estimate the gyro-free roll angle and roll rotation rate under the harsh environment. 
MEMS gyro is commonly used in the rolling angle measurement of missile, unmanned aerial vehicle, and other unmanned attitude measurement. In the flight environment of high axial rotary speed and high overload, this kind of system has some problems, such as performance degradation or even failure after overload and initial alignment difficulty, which makes it impossible to continue attitude estimation. Therefore, in recent years, more and more systems use geomagnetic information to obtain roll angle. The rolling angle measurement scheme using geomagnetic information has the advantages of small space occupation, fast response speed, good antihigh overload performance, no error accumulation over time, low cost, etc. $[1,2]$ and can be normally solved under high overload and high dynamic flight environment. However, due to the magnetic field characteristics, its roll rotation angle is easily interfered by the projectile body magnetic field, and the accuracy of roll rotation is closely related to the measurement accuracy of geomagnetic field.

In order to estimate the axial speed of the projectile by using magnetic measurement information, time-frequency domain analysis methods such as STFT (Short-time-Fourier-transform) [3-5], differential filtering [6], zero pass detection $[7,8]$, and deformation Kalman filtering algorithm combined with peak detection are currently employed [9], but these methods all have their own defects. For example, the STFT estimation method uses Fourier transform to estimate the angular rate. In the case of high dynamics, there is a contradiction between the accuracy and real-time performance of this method in estimating the roll angular rate, that is, the longer the interval time, the higher the estimation accuracy, but the worse the real-time performance. The shorter the interval time, the better the real-time estimation, but the lower the estimation frequency accuracy. Differential filtering method is to get the angular rate information by difference of magnetic measurement signal. The error of this method is large, and the angular rate error obtained by difference will be larger when there is a measurement error of magnetic signal. After low-pass filtering [10], the estimation error can be reduced, but the estimation result will be delayed. Similarly, the rate of change obtained directly by magnetic signals is also greatly affected by measurement errors. The detection method of zero crossing is to obtain angular rate information by measuring the time of each zero crossing through sinusoidal magnetic measurement signal. However, data updating rate of this method is different under the influence of rotational speed, that is, when the axial rotational speed is fast, the angular rate information updates quickly; when the axial rotational speed is too slow, the angular rate information updates slowly, and the information update rate cannot be determined.

$\mathrm{n}$ addition, for only using the magnetometer to obtain the angular rate and other attitude information, also through the establishment of the projectile dynamics model, Li et al. introduces a kind of gyro angular rate estimation method [11], Natanson and others introduce a three-axis magnetometer measurement spacecraft attitude and angular rate RTSF (real-time) sequential filtering method, and $\mathrm{Ma}$ and $\mathrm{Xu}$ put forward improved real-time sequential filtering method (IRTSF) [12]. Sabzevari et al. use the magnetometer $[13,14]$ to estimate the attitude in vehicle dynamics model is established on the basis of calculating. However, in the process of high-speed flight, the force and torque, including aerodynamic force, subjected to the projectile are very complex; therefore, accurate dynamic model cannot be established. Hu et al. proposed a new filtering method based on UKF, which is suitable for combined inertial navigation and GPS measurement [15]. On the contrary, the change rate of roll angle obtained directly by magnetic signal is greatly affected by the measurement error [16]. Therefore, a simple, fast, practical, and high accuracy algorithm is needed to estimate the real-time roll angle and roll angle rate of the rotating projectile.

In this paper, the roll angle and angular rate estimation based on geomagnetic information are estimated when the projectile's torque is unknown. Firstly, the kinematics model is established according to the motion characteristics of the projectile. Based on the accurate estimation of projectile roll angle and roll angle rate by the Kalman filter, in view of the dynamic characteristics of projectile motion and high speed, appropriate forgetting factor is added for strong tracking, to change the measurement noise and system noise in real time improve the adaptive ability of the algorithm [17]. Thus, the attitude information of roll angle and roll angle rate necessary for guidance and control of guided munitions under bad onboard environment is obtained.

\section{Establish Coordinate System}

Geomagnetic attitude measurement system is mainly based on the information of geomagnetic field. Although geomagnetic field is a global long-term changing magnetic field, it changes slowly and is almost constant in a very short period of time compared with the rapid launch of ammunition. Therefore, geomagnetic information can be widely used in aviation, aerospace, navigation, and other fields. The three-axis magnetic sensor can measure the three components of geomagnetic field in each position in real time. It has the advantages of not accumulating errors with time, low cost, and high antioverload performance. Using the three components of the geomagnetic field at the initial time of launch and the information of the three components of the geomagnetic field at any time during the projectile's flight, the attitude angle of the projectile can be obtained through the transformation of the coordinate system $[18,19]$. After that, roll angle and roll rotation rate can be further estimated, so the first thing is to establish the coordinate system.

In order to obtain the motion attitude angle of the projectile body relative to the initial moment, two coordinate systems need to be established, namely, the launching coordinate system $f$ system $\left(O-X_{f} Y_{f} Z_{f}\right)$ and the projectile body coordinate system $b$ system $\left(O^{\prime}-X_{b} Y_{b} Z_{b}\right)$. The launch as a benchmark coordinate system belongs to the static coordinate system; in order to determine the initial position of the projectile, the origin is located at the centroid at the launch site, the $X$-axis is from origin to destination, $Y$-axis vertically points inside the centroid transverse section, $Z$ axis within the centroid transverse section pointing to the 
right level, and coordinates measured three component $\left[\begin{array}{lll}H_{x}^{f} & H_{y}^{f} & H_{z}^{f}\end{array}\right]^{T}$ is the initial three-component magnetic field. The missile system belongs to the moving coordinate system, which is used to represent the motion attitude of the projectile at any moment of its flight relative to the initial launch time. Its origin is located in the center of mass of the projectile, and it moves with the projectile's flight motion. The $X$-axis is along the direction of the projectile axis, and the $Y$-axis and $Z$-axis are located in the transverse section of the center of mass and rotate with the projectile around the $X$-axis The geomagneticfield three components $\left[\begin{array}{lll}H_{x}^{b} & H_{y}^{b} & H_{z}^{b}\end{array}\right]^{T}$ measured in the missile coordinate system is the three-axis magnetic field value at each position and attitude during the flight of the projectile. Both coordinate systems satisfy the right-hand rule, as shown in Figure 1.

The transformation matrix of the launching system to the projectile system can be obtained according to the projection relation and rotation mode, and the rotation matrix results are different with different rotation orders. In this paper, rotation is carried out according to the rotation mode of 231, that is, the yaw angle rotation matrix $C_{\psi}$ is obtained by first rotating about the $Y$-axis. Then, rotate about the $Z$-axis to get the pitch angle rotation matrix $C_{\theta}$. Finally, rotate about the $X$-axis to get the roll rotation matrix $C_{\gamma}$. The details are shown below:

$$
\begin{aligned}
& \mathbf{C}_{\psi}=\left(\begin{array}{ccc}
\cos \psi & 0 & -\sin \psi \\
0 & 1 & 0 \\
\sin \psi & 0 & \cos \psi
\end{array}\right), \\
& \mathbf{C}_{\theta}=\left(\begin{array}{ccc}
\cos \theta & \sin \theta & 0 \\
-\sin \theta & \cos \theta & 0 \\
0 & 0 & 1
\end{array}\right), \\
& \mathbf{C}_{\gamma}=\left(\begin{array}{ccc}
1 & 0 & 0 \\
0 & \cos \gamma & \sin \gamma \\
0 & -\sin \gamma & \cos \gamma
\end{array}\right) .
\end{aligned}
$$

Finally, according to the rotation order of $Y-Z-X$, multiply the three rotation matrices to obtain the rotation matrix $\mathbf{C}_{f}^{b}$ of the launching system to the projectile system as follows:

$$
\begin{aligned}
C_{f}^{b} & =C_{x} C_{z} C_{y}=C_{\gamma} C_{\theta} C_{\psi} \\
& =\left(\begin{array}{ccc}
\cos \psi \cos \theta & \sin \theta & -\sin \psi \cos \theta \\
\sin \psi \sin \gamma-\cos \psi \sin \theta \cos \gamma & \cos \theta \cos \gamma & \cos \psi \sin \gamma+\sin \psi \sin \theta \cos \gamma \\
\sin \psi \cos \gamma+\cos \psi \sin \theta \sin \gamma & -\cos \theta \sin \gamma & \cos \psi \cos \gamma-\sin \psi \sin \theta \sin \gamma
\end{array}\right) .
\end{aligned}
$$

Thus, the relationship between the three components $\mathbf{H}^{f}$ of the initial magnetic field in the emission system and the three components $\mathbf{H}^{b}$ of the magnetic field in the carrier coordinate system can be obtained:

$$
\begin{aligned}
\mathbf{H}^{b} & =C_{n}^{b} \mathbf{H}^{f}, \\
\mathbf{H}^{b} & =\left[\begin{array}{lll}
H_{x}^{b} & H_{y}^{b} & H_{z}^{b}
\end{array}\right]^{T}, \\
\mathbf{H}^{f} & =\left[\begin{array}{lll}
H_{x}^{f} & H_{y}^{f} & H_{z}^{f}
\end{array}\right]^{T} .
\end{aligned}
$$

\section{Estimation Algorithm}

3.1. Magnetic Information to Solve the Roll. From the above section, it can be seen that the attitude angle of the projectile body can be solved by using geomagnetic field information. When the three components of the projectile body's magnetic field and the three components of the projectile body's magnetic field are known, one of the attitude angles must be known to solve the other two attitude angles. Due to the fact that the yaw angle of the guided munitions, such as the rotary bomb, is small in the trajectory fire plane during flight, the yaw angle can be set as 0 , and the following formula can be obtained:

$$
\left[\begin{array}{c}
H_{x}^{b} \\
H_{y}^{b} \\
H_{z}^{b}
\end{array}\right]=\left[\begin{array}{ccc}
\cos \theta & \sin \theta & 0 \\
-\sin \theta \cos \gamma & \cos \theta \cos \gamma & \sin \gamma \\
\sin \theta \sin \gamma & -\cos \theta \sin \gamma & \cos \gamma
\end{array}\right]\left[\begin{array}{c}
H_{x}^{f} \\
H_{y}^{f} \\
H_{z}^{f}
\end{array}\right] .
$$

Thus, the formula of pitch angle and roll angle of the projectile can be obtained as follows:

$$
\begin{aligned}
& \theta=\arctan \left(\frac{H_{x}^{n}}{H_{z}^{n}}\right)-\arcsin \left[\frac{H_{x}^{b}}{\sqrt{\left(H_{x}^{n}\right)^{2}+\left(H_{z}^{n}\right)^{2}}} \frac{H_{x}^{b}}{\sqrt{\left(H_{x}^{n}\right)^{2}+\left(H_{z}^{n}\right)^{2}}}\right], \\
& \gamma=\arctan \left[\frac{\left(H_{x}^{n} \sin \theta+H_{z}^{n} \cos \theta\right) H_{y}^{b}-H_{y}^{n} H_{z}^{b}}{\left(H_{x}^{n} \sin \theta+H_{z}^{n} \cos \theta\right) H_{z}^{b}+H_{y}^{n} H_{y}^{b}}\right] .
\end{aligned}
$$

3.2. Estimation of Roll Angle and Roll Rate. In the kinematics of the projectile's external trajectory, the attenuation law of the axial rotational speed of the projectile's external ballistic trajectory can be obtained according to the Roguery formula [20]:

$$
\dot{\gamma}=\dot{\gamma}_{0} \exp \left(-0.075 k \frac{L D^{3}}{A} t\right)
$$


where $L$ is the projectile length, $D$ is the projectile diameter, $A$ is the moment of inertia of the projectile pole, and $k$ is the coefficient.

Thus, we can know that the axial rotation speed $\omega$ of the projectile decreases exponentially with time $t$. The rotational axial rotational speed of the projectile conforms to the flexible formula in the uncontrolled-free flight stage or in the single control period, so the roll angular velocity of the projectile can be regarded as the change of uniform deceleration in a short time. According to this characteristic, a quadratic kinematics equation can be established as the equation of state:

$$
\gamma(t)=\frac{1}{2} \mathrm{at}^{2}+\mathrm{bt}+c,
$$

where $a, b$, and $c$ are constants.

Suppose in the flight process of the rotating projectile, the rotational angle, rotational angle rate, and rotational angle acceleration at time $T$ are $\gamma(t), \omega(t)$, and $a(t)$, respectively. From the above formula, it can be seen that the angular velocity in a short time is uniform, in other words, the roll angle plus acceleration is a random variable, and the roll angle acceleration $a(t)$ is driven by white noise. If the white noise is $j(t)$, the mean value of the system noise is zero and the covariance matrix is $Q$, and the following kinematic relationship can be obtained:

$$
\begin{gathered}
\dot{\gamma}(t)=\omega(t), \\
\dot{\omega}(t)=a(t), \\
\dot{a}(t)=j(t),
\end{gathered}
$$

where $E\left[j_{t}\right]=0$ and $E\left[j_{t} j_{k}^{T}\right]=Q_{t} \delta_{\text {tk }}$.

Then, the state equation of the model can be written as follows:

$$
\dot{X}(t)=F X(t)+G j(t),
$$

where

$$
\begin{aligned}
X(t) & =\left[\begin{array}{l}
\gamma(t) \\
\omega(t) \\
a(t)
\end{array}\right], \\
F & =\left[\begin{array}{lll}
0 & 1 & 0 \\
0 & 0 & 1 \\
0 & 0 & 0
\end{array}\right], \\
G & =\left[\begin{array}{l}
0 \\
0 \\
1
\end{array}\right] .
\end{aligned}
$$

After that, the roll angle calculated by the magnetic measurement system is taken as the observation value, and the measurement model can be obtained as follows:

$$
Z_{t}=H X_{t}+V_{t},
$$

where $H$ is the measurement matrix:

$$
H=\left[\begin{array}{lll}
1 & 0 & 0
\end{array}\right] .
$$

$V_{\mathrm{t}}$ is the measurement noise, the mean value is zero, and the covariance matrix is $R$. The equivalent of $E\left[V_{\mathrm{t}}\right]=0$ and $E\left[V_{\mathrm{t}} V_{k}^{T}\right]=R_{t} \delta_{\mathrm{tk}}$.

When the sampling period is $T_{\mathrm{s}}$, the model is discretized and the results are as follows:

$$
X_{k}=\phi_{k / k-1} X_{k-1}+\int_{k-1}^{k} \phi(k, \tau) G j(\tau) \mathrm{d} \tau,
$$

where $\phi_{k / k-1}=I+F T_{s}+\left(T_{S}^{2} / 2 !\right) F^{2}+\left(T_{S}^{3} / 3 !\right) F^{3}+\cdots$.

When the sampling interval is small, that is, the sampling frequency is large, the higher order term can be omitted, and the one-step transfer matrix is

$$
\phi_{t / t-1}=\left[\begin{array}{ccc}
1 & T_{s} & 0 \\
0 & 1 & T_{s} \\
0 & 0 & 1
\end{array}\right] .
$$

At the same time, the system noise driving array can be obtained as follows:

$$
\Gamma=\left[0 \frac{T_{s}^{2}}{2} T_{s}\right]^{T} .
$$

Then, the final equation of state of the system is

$$
X_{k}=\phi_{k / k-1} X_{k-1}+\Gamma j_{k-1}
$$

where $X_{k}=\left[\begin{array}{lll}\gamma_{k} & \omega_{k} & a_{k}\end{array}\right]^{T}$.

In the process of rotating projectile launch, there are abrupt changes in the projectile roll angular rate, which do not conform to the model setting of "short-time uniform acceleration," and thus the model is not accurate enough.Therefore, for the estimation of roll angle and roll angle rate, the measurement noise and system noise of the model should be appropriately modified to improve the weight of new information and reduce the interference of the previous estimation results to the current time estimation.

Assuming that, in the angular rate mutation stage, the variance matrix of measurement noise $V(k)$ and system noise $W(k)$ are, respectively,

$$
\begin{aligned}
E\left[V_{k} V_{j}^{T}\right] & =s^{N-k} R_{k} \delta_{\mathrm{kj}}, \\
E\left[W_{k} W_{j}^{T}\right] & =s^{N-k+1} Q_{k} \delta_{\mathrm{kj}},
\end{aligned}
$$




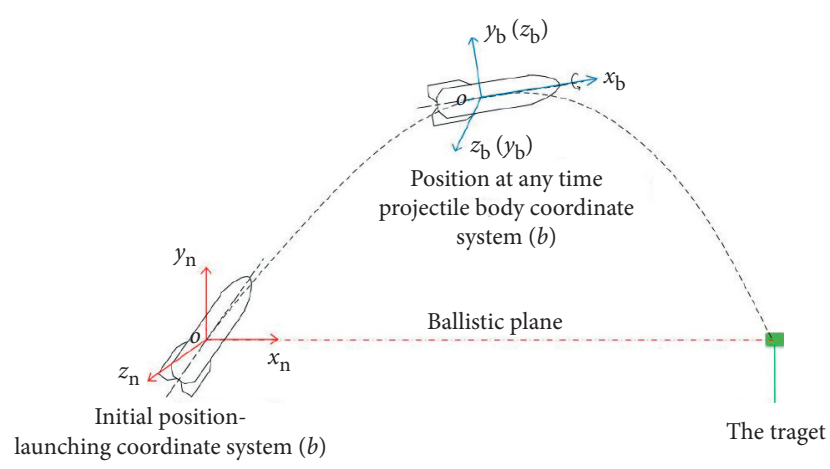

FIgURE 1: The relationship between the two coordinates.

where $k$ and $j$ are both a certain moment in time series $N$. It can be seen that when $s$ is 1 , the noise variance matrix does not change. When $s$ is greater than 1 , the noise variance matrix increases. According to the case that the model estimation error increases when the angular rate changes, $s$ should be a real number slightly greater than 1 . Thus, a new one-step prediction mean square error matrix and gain matrix are

$$
\begin{gathered}
P_{k / k-1}=\phi_{k / k-1} P_{k-1} \phi_{k / k-1}^{T}+\Gamma_{k-1} s^{N-k} Q_{k-1} \Gamma_{k-1}^{T}, \\
K_{k}=P_{k / k-1} H_{k}^{T}\left(H_{k} P_{k / k-1} H_{k}^{T}+s^{N-k} R_{k}\right)^{-1} .
\end{gathered}
$$

By multiplying the one-step prediction mean square error of the above equation by $s^{-(N-k)}$, we can obtain

$$
s^{-(N-k)} P_{k / k-1}=\phi_{k / k-1} s^{-(N-k)} P_{k-1} \phi_{k / k-1}^{T}+\Gamma_{k-1} Q_{k-1} \Gamma_{k-1}^{T} \text {. }
$$

Assume that

$$
P_{k / k-1}^{*}=\phi_{k / k-1}\left(s P_{k-1}^{*}\right) \phi_{k / k-1}^{T}+\Gamma_{k-1} Q_{k-1} \Gamma_{k-1}^{T} .
$$

Similarly, multiply the left and right sides of the estimated mean square error by $s^{-(N-k)}$ to simplify and arrange the final model equation as follows:

$$
\begin{aligned}
\widehat{X}_{k / k-1}^{*} & =\phi_{k / k-1} * \widehat{X}_{k-1}^{*}, \\
P_{k / k-1}^{*} & =\phi_{k / k-1}\left(\mathrm{sP}_{k-1}^{*}\right) \phi_{k / k-1}^{T}+\Gamma_{k-1} Q_{k-1} \Gamma_{k-1}^{T}, \\
K_{k}^{*} & =P_{k / k-1}^{*} H_{k}^{T}\left(H_{k} P_{k / k-1}^{*} H_{k}^{T}+R_{k}\right)^{-1}, \\
\widehat{X}_{k}^{*} & =\widehat{X}_{k / k-1}^{*}+K_{k}^{*}\left(Z_{k}-H_{k} \widehat{X}_{k / k-1}^{*}\right), \\
P_{k}^{*} & =\left(I-K_{k} H_{k}\right) P_{k / k-1}^{*},
\end{aligned}
$$

where $\widehat{X}_{k-1}^{*}, \widehat{X}_{k}^{*}, \widehat{X}_{k / k-1}^{*}$, and $K_{k}^{*}$, respectively, represent the estimated value at time $k-1$ and time $k$ as well as the state prediction and gain at time $k$, and $s$ is the forgetting factor.

It can be seen that the simplified filtering model can only be multiplied by a forgetting factor before the estimated mean square error at the previous moment.

\section{Results and Discussion}

4.1. Validation of Simulation Data. According to the above model, the initial value is given for simulation verification.

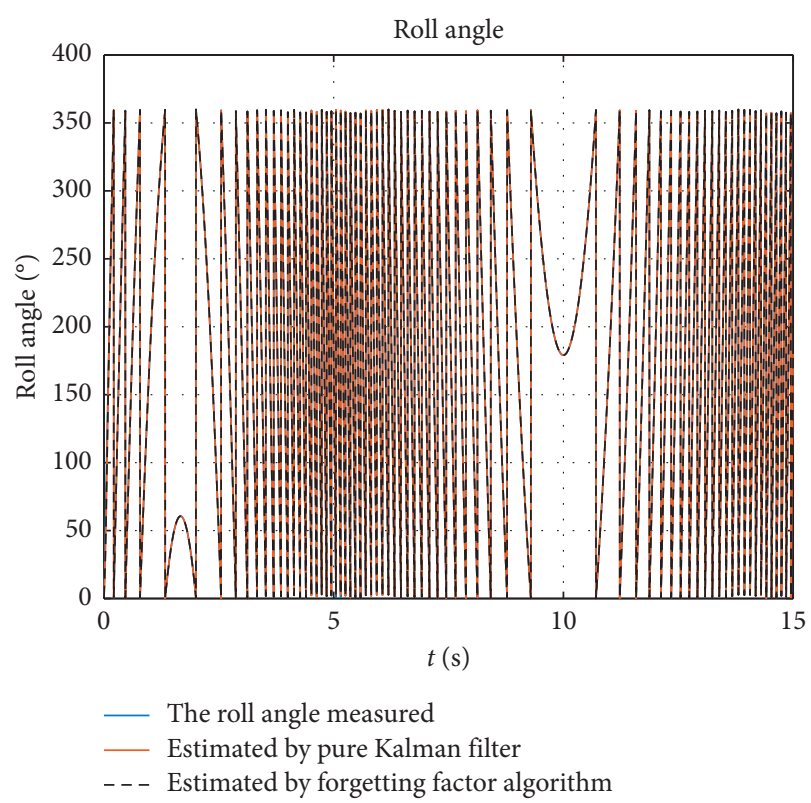

FIgURE 2: The roll angles of the generated and the estimated.

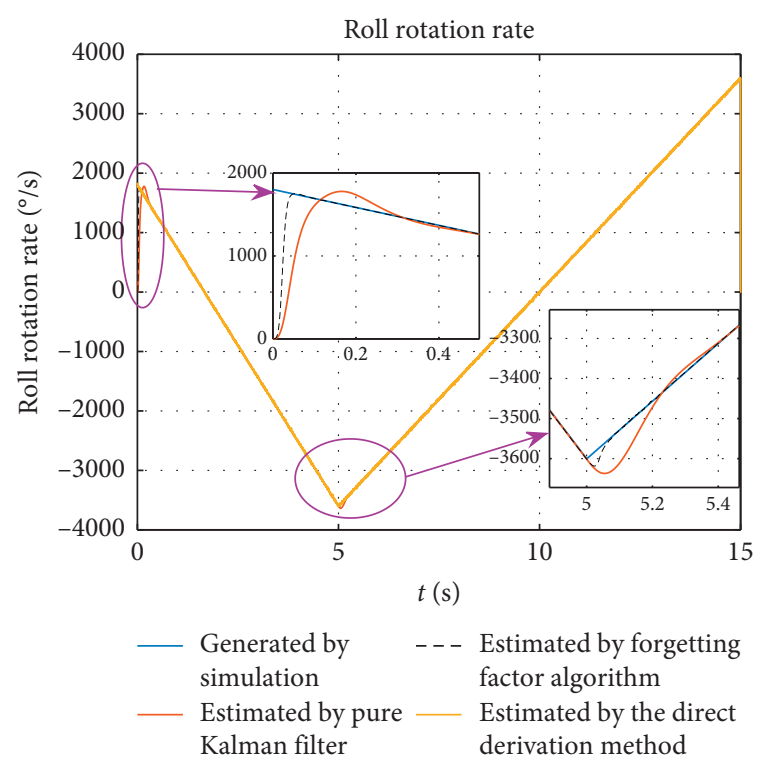

Figure 3: The roll angle rate of the generated and the estimated.

First, a set of roll angle and roll angle rate data with angular rate mutation are generated by simulation, and appropriate measurement error is added, and then the algorithm is used for estimation. $Q=22, R=0.012$, and $s=1.2$ are set in this group of simulation data. The estimated results and the original generated data are shown in the figure below.

In the above simulation results, it can be concluded from Figures 2 and 3 that the estimation algorithm can simultaneously estimate the roll angle and roll angle rate of the projectile. From Figures 3-5, it can be seen that the angular rate mutation exists at $0 \mathrm{~s}$ and $5 \mathrm{~s}$. It can be seen from Figures 4 and 5 that the error of the roll angle and roll angle rate estimated by the Kalman filter is large due to the 


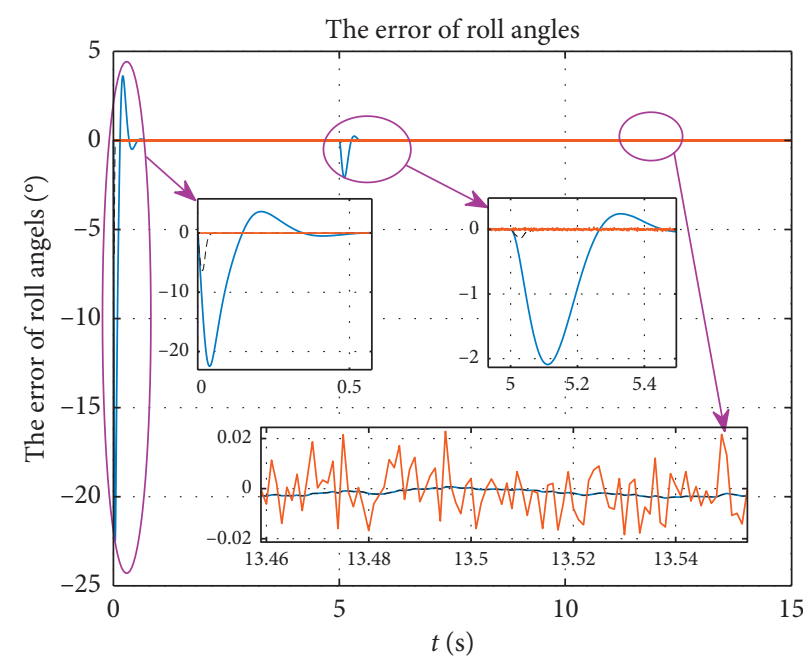

Estimation error of pure Kalman filter

- - - Estimation error with forgetting factor algorithm

_ Error of measurement

Figure 4: The error of roll angles.

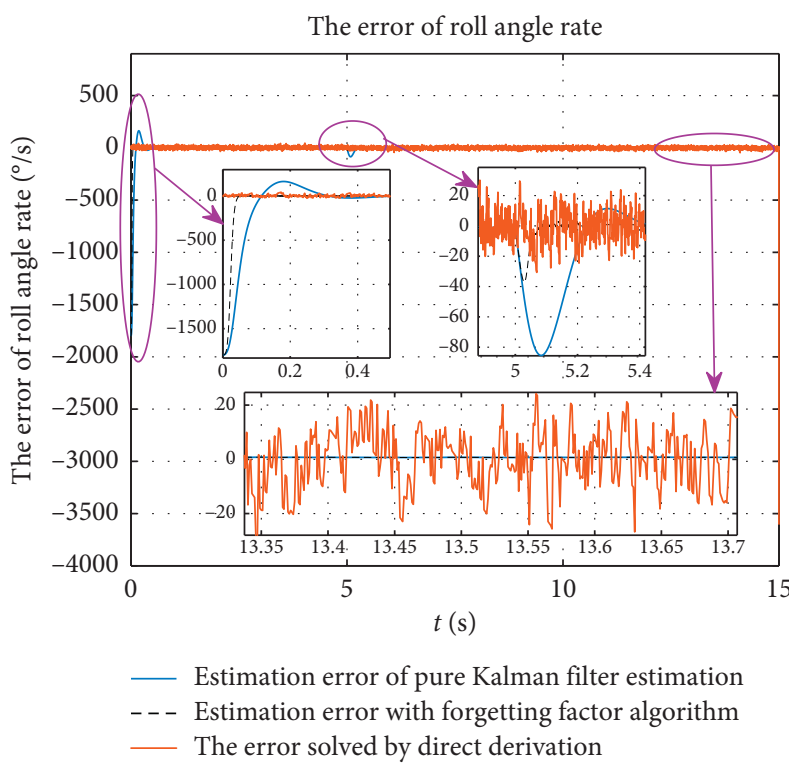

Figure 5: The error of the roll angle rate.

TABLE 1: The estimation error of the algorithm in the angular rate mutation stage.

\begin{tabular}{|c|c|c|c|c|}
\hline \multirow{2}{*}{$\begin{array}{l}\text { The stage of angular } \\
\text { rate mutation }\end{array}$} & \multicolumn{2}{|c|}{ Mean of roll angle error $\left({ }^{\circ}\right)$} & \multicolumn{2}{|c|}{ Mean of roll angle rate error $(\%)$} \\
\hline & $\begin{array}{l}\text { Estimated by pure } \\
\text { Kalman filter }\end{array}$ & $\begin{array}{l}\text { Estimated by the algorithm } \\
\text { with forgetting factor }\end{array}$ & $\begin{array}{l}\text { Estimated by pure } \\
\text { Kalman filter }\end{array}$ & $\begin{array}{l}\text { Estimated by the algorithm } \\
\text { with forgetting factor }\end{array}$ \\
\hline $0 \mathrm{~s}-0.55 \mathrm{~s}$ & -2.3443 & -0.2431 & -139.15 & -77.3721 \\
\hline $5 s-5.6 s$ & -0.4660 & -0.0080 & -16.8456 & -1.6650 \\
\hline
\end{tabular}

constraint of the estimation model, while the error of the algorithm estimated by adding the forgetting factor will be greatly reduced. The error of roll angle and roll angle rate at the angular rate mutation is shown in the table below.
It can be seen from Table 1 that, in the angular rate mutation stage, compared with the pure Kalman filter estimation, the estimation accuracy of the algorithm with the forgetting factor is improved by an order of magnitude, and 
the accuracy of the roll rotation rate is increased by more than 4 times. It can be seen from Figure 5 that, in the stationary phase, the accuracy of the roll rotation rate estimated by the algorithm is 10 times higher than that obtained by direct derivation. The validity of the algorithm is verified.

4.2. Validation of Semiphysical Turntable Data. After the algorithm is verified, it is verified according to the data of the magnetic measurement system. With the three-axis magnetometer HMC1053 produced by Honeywell company as the only attitude sensing chip and the STM 32 single chip microcomputer produced by ST Company as the controller, the control circuit was designed to constitute the magnetic attitude measurement system [21, 22], and the magnetic measurement system was fixed on the three-axis highprecision flight simulation turntable, as shown in Figure 6. The control table rotates around the $X$-axis, $Y$-axis, and $Z$ axis, respectively, and the roll angle is calculated by using the magnetic measurement system, and the roll angle and the roll rotation rate are optimized and estimated by taking the magnetic measurement as the observation data.

In this experiment, the gyroscope is saturated and it is impossible to further estimate the carrier roll angle, so the gyro information is only used to estimate the roll angle rate estimated by the algorithm in the later stage.

To simulate the flight state of the projectile body under the maneuvering condition, the flight turntable was accelerated uniformly around the $X$-axis to $5 \mathrm{r} / \mathrm{s}$ and then decelerated uniformly to $0 \mathrm{r} / \mathrm{s}$ after maintaining $21 \mathrm{~s}$, as shown in Figure 7. In the model parameter setting, $Q=22$, $R=0.52$, and $s=1.03$, and the simulation results are shown in the figures.

The estimated roll angle and roll rotation rate are shown in Figures 7 and 8. It can be seen from Figure 8 that, at the angular rate mutation of $1 \mathrm{~s}, 3 \mathrm{~s}$, and $23 \mathrm{~s}$, the roll angle and roll rate estimated by the pure Kalman filter have large error. However, the error estimated by adding forgetting factor algorithm is significantly smaller, as shown in Table 2.

As can be seen from Table 2, in the angular rate mutation stage, compared with the estimation result of the pure Kalman filter, the roll angle accuracy estimated by the algorithm with the forgetting factor is improved by an order of magnitude. The accuracy of the estimated roll rotation rate is improved by more than 4 times. It can also be seen from Figures 9 and 10 that, in the stationary phase, the roll angle error estimated by the algorithm is within $2^{\circ}$, which is more than twice as accurate as the roll error of $5^{\circ}$ measured. The error of the roll rotation rate estimated by the algorithm is within $5 \%$, which improves the accuracy by an order of magnitude compared with the error of the roll rotation rate obtained by direct derivation of $50 \%$, which verifies the feasibility of the algorithm and the system.

4.3. Verification of Bomb Test Data. The feasibility of the algorithm is verified by theoretical analysis and turntable semiphysical simulation test. Now, the sensor data

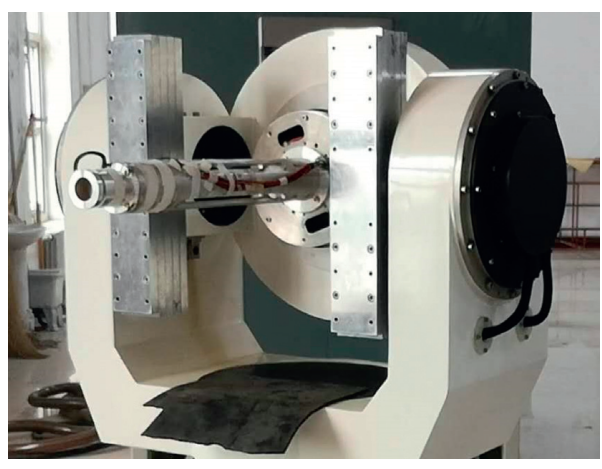

Figure 6: Three-axis high-precision flight simulation turntable.

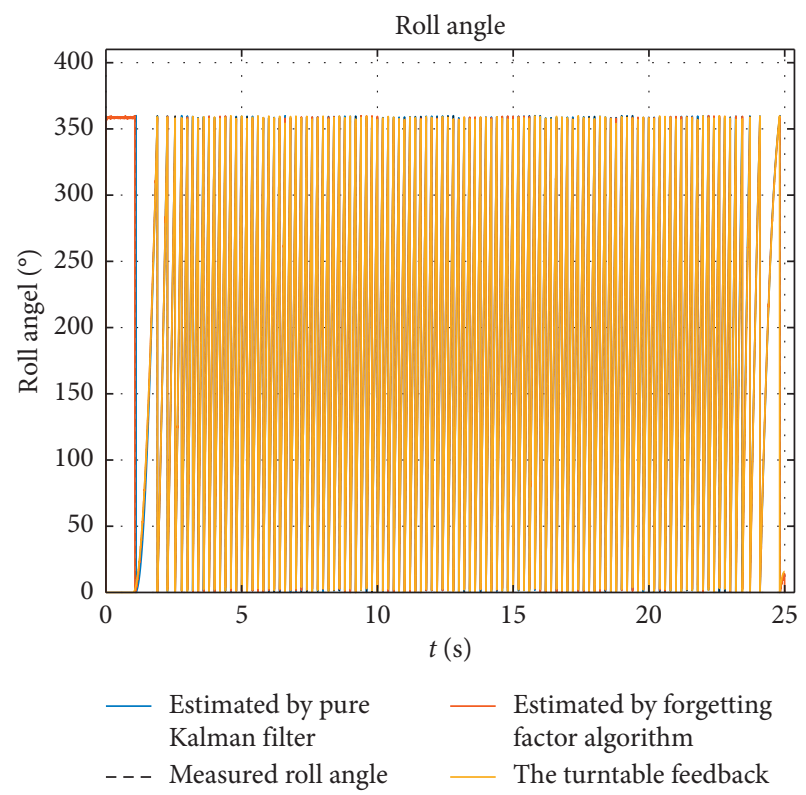

FIgURE 7: The roll angles of turntable feedback and algorithm estimation.

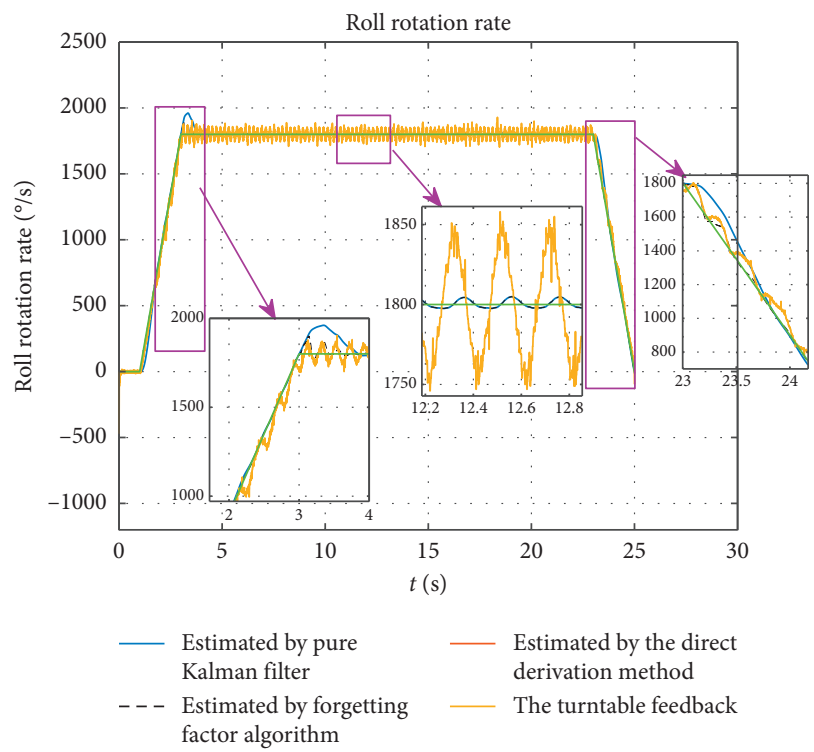

FIgURE 8: The roll angles rate of turntable feedback and algorithm estimation. 
TABLE 2: The estimation error of the algorithm in the angular rate mutation stage.

\begin{tabular}{|c|c|c|c|c|}
\hline \multirow[b]{2}{*}{$\begin{array}{l}\text { The stage of angular rate } \\
\text { mutation }\end{array}$} & \multicolumn{2}{|c|}{ Mean of roll angle error $\left({ }^{\circ}\right)$} & \multicolumn{2}{|c|}{ Mean of roll angle rate error $(\% / \mathrm{s})$} \\
\hline & $\begin{array}{l}\text { Estimated by pure Kalman } \\
\text { filter }\end{array}$ & $\begin{array}{l}\text { Estimated by the } \\
\text { algorithm with forgetting } \\
\text { factor }\end{array}$ & $\begin{array}{l}\text { Estimated by } \\
\text { pure } \\
\text { Kalman filter }\end{array}$ & $\begin{array}{l}\text { Estimated by the } \\
\text { algorithm } \\
\text { with forgetting factor }\end{array}$ \\
\hline $1 \mathrm{~s}-2 \mathrm{~s}$ & -8.2261 & -0.5716 & -74.5018 & -4.1254 \\
\hline $3 s-4 s$ & 6.6852 & -0.6276 & 77.6076 & 15.4660 \\
\hline $23 s-24 s$ & 7.3149 & -0.0166 & 78.4181 & 16.0643 \\
\hline
\end{tabular}

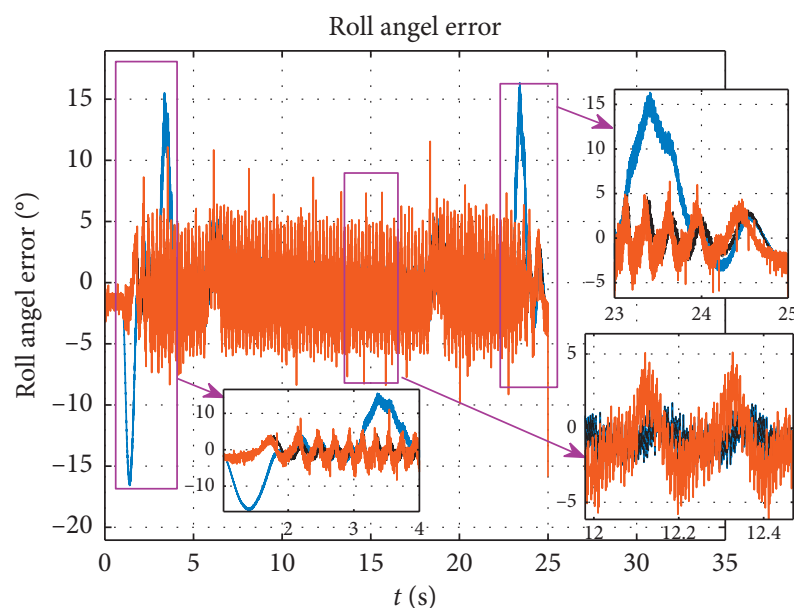

_ Estimated by pure Kalman filter

- - - Estimated by forgetting factor algorithm

— Error of roll angel measured

Figure 9: The error of the roll angle.

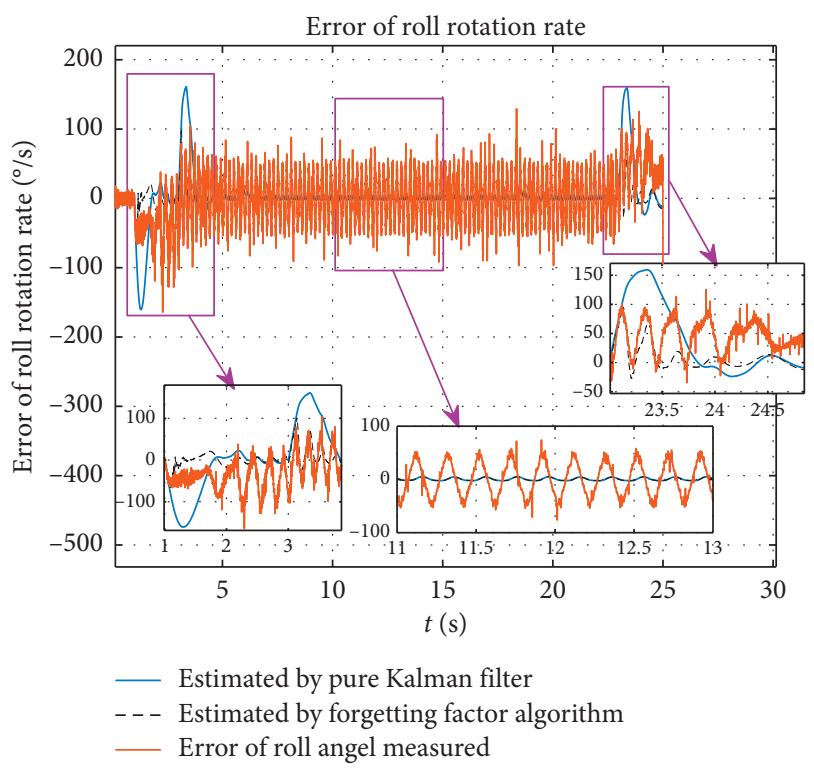

Figure 10: The error of the roll angle rate.

collected from the ballistic flight test of the range is used for further verification. A magnetic measuring system and an axial MEMS gyro are installed in the test projectile body. The roll rotation rate of the gyro output is taken as a reference to verify the accuracy of the estimated roll

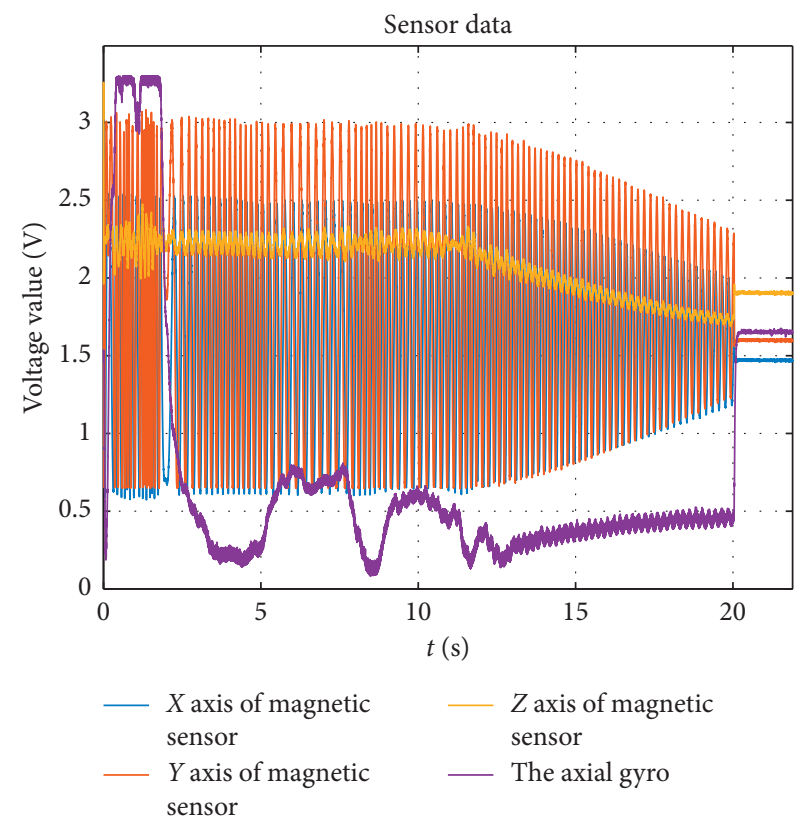

FIgURE 11: Sensor data of magnetic and gyro.

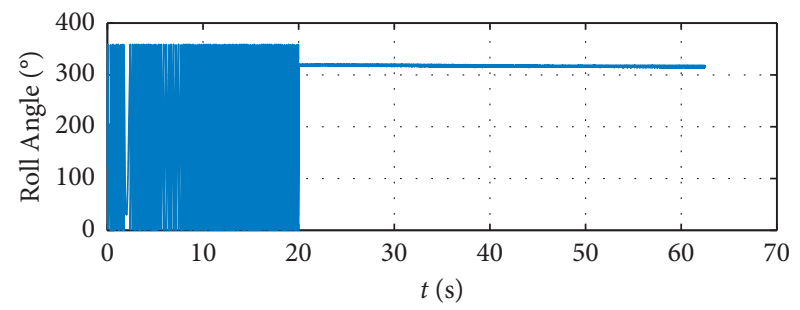

(a)

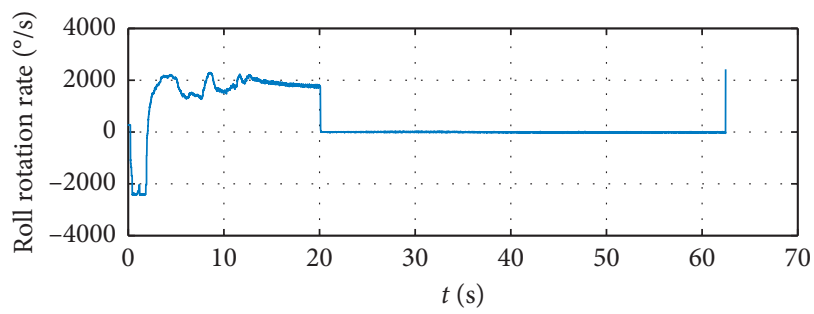

(b)

Figure 12: The system calculates the roll angle and the angular rate of the gyro output. (a) The role angle that system calculates. (b) Rotation rate of gyro type.

rotation and roll rotation rate calculated by using only the magnetometer data. The test results are shown in the figures. 


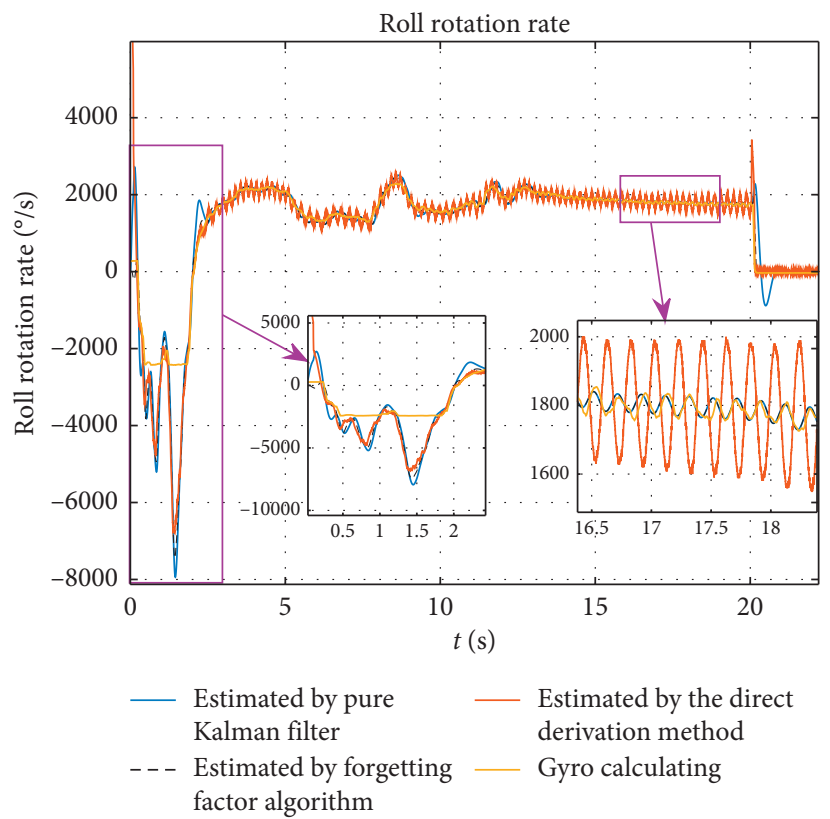

Figure 13: The roll angle rate that the system calculates and the gyro output.

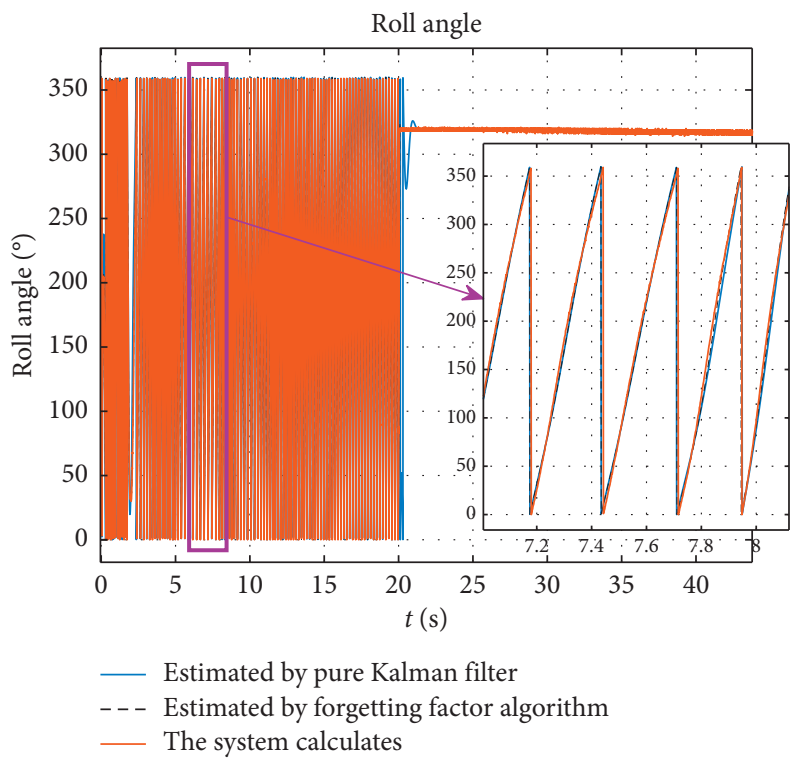

FIgURE 14: The roll angle that the system calculates and the gyro output.

The figure above shows the system output and algorithm estimation results of the bomb test. It can be seen from Figure 11 that the effective flight time of the ballistic test is $20 \mathrm{~s}$. It can be seen from Figures 12 and 13 that the gyro is saturated during flight and cannot normally calculate the roll rotation rate. However, the roll rotation rate estimated by the algorithm makes up for this defect. The roll angle and roll rotation rate estimated by the algorithm are shown in Figures 13 and 14. Figure 14 shows that the roll angle estimated by the algorithm is better than the linearity of the roll angle calculated directly by the system, which indicates that the roll angle estimated by the algorithm compensates some errors caused by the system measurement. Figure 13 shows that the roll rotation rate estimated by the algorithm compensates for the error caused by gyro saturation in the first two seconds. In the stationary phase, the accuracy of the roll rotation rate estimated by the algorithm is 6 times higher than that obtained by direct derivation. Figure 15 shows that, in the angular rate mutation stage, the roll angular rate estimated by forgetting factor reduces the error caused by pure Kalman filter estimation, and the mean value of the estimated error caused by angular rate mutation is shown in the table.

It can be seen from Table 3 that the angular rate at $11 \mathrm{~s}$ does not change much, so the effect of the algorithm with the forgetting factor is not obvious. However, in other abrupt changes of angular rates, the accuracy of the algorithm with 


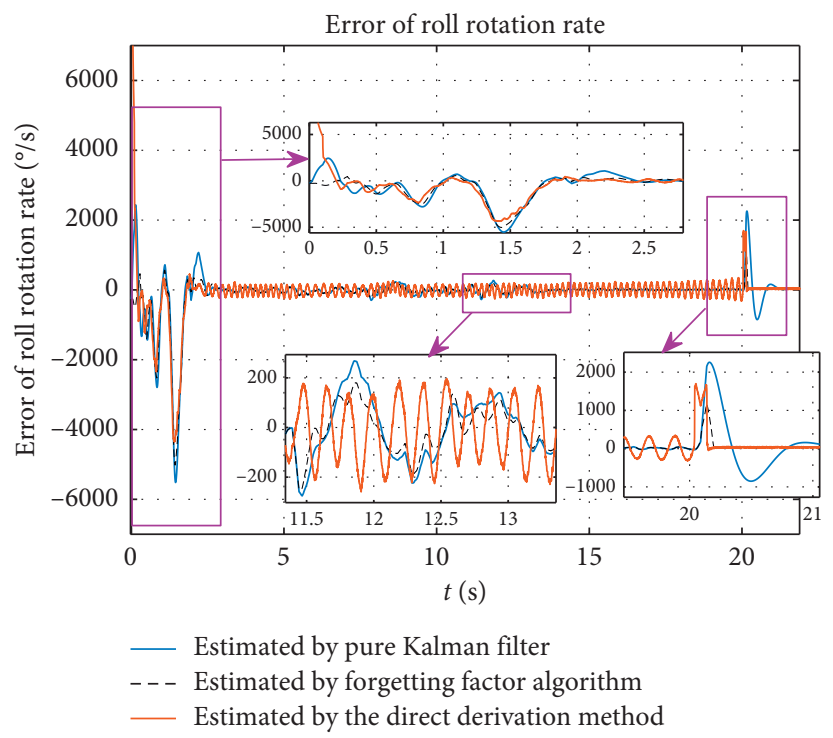

FIgURE 15: The error of roll angle rate that the system calculates and the gyro output.

TABLE 3: The estimation error of the algorithm in the angular rate mutation stage.

\begin{tabular}{lcc}
\hline \multirow{2}{*}{ The stage of angular rate mutation } & \multicolumn{2}{c}{ Mean of roll angle rate error $(/ \mathrm{s})$} \\
& Estimated by pure Kalman filter & Estimated by the algorithm with forgetting factor \\
\hline $0 \mathrm{~s}-0.2 \mathrm{~s}$ & 1450.5 & -318.9104 \\
$2 \mathrm{~s}-2.5 \mathrm{~s}$ & 570.6310 & 144.0300 \\
$11.5 \mathrm{~s}-12 \mathrm{~s}$ & 81.3683 & 62.8377 \\
$20 \mathrm{~s}-20.4 \mathrm{~s}$ & 824.1634 & 208.4635 \\
\hline
\end{tabular}

the forgetting factor is more than 4 times higher than that of the pure Kalman filter.

\section{Conclusions}

In this paper, we propose a method to estimate the roll angle and roll angle rate of a projectile by using only the magnetic field information provided by a triaxial magnetometer, and a real-time estimation algorithm based on the Kalman filter with appropriate forgetting factor is proposed. This method solves the problem that the projectile roll angle and roll angle rate cannot be obtained due to MEMS gyro overload and degradation under the flight condition of high spin and high overload. The Kalman filter estimation algorithm with the oblivion factor is able to significantly reduce the error caused by estimation delay under high dynamic conditions.

Through the above analysis and semiphysical simulation test, it can be concluded that the algorithm can estimate the roll angle and roll angle rate of the carrier in real time and quickly. The experimental results show that the algorithm with the forgetting factor reduces the influence of magnetic sensor measurement error on the accuracy of roll angle and improves the accuracy of roll angle by one time. The experimental results show that the error of the roll rotation rate estimated by this algorithm is within $5 \%$, and the accuracy is 6 times higher than that obtained by direct derivation. In the angular rate mutation phase, compared with the pure Kalman filter estimation algorithm, the accuracy of the roll angle estimated by the algorithm that the Kalman filter with the forgetting factor is improved by an order of magnitude, and the accuracy of the roll angle rate is improved by at least four times, which can meet the requirements of the projectile roll angle and roll angle rate of the guidance and control system of general rotating bombs.

\section{Data Availability}

The data used to support the findings of this study are available from the corresponding author upon request.

\section{Disclosure}

Lizhen Gao and Yingying Zhang are co-first authors of this article.

\section{Conflicts of Interest}

The authors declare that they have no conflicts of interest regarding the publication of this paper.

\section{Acknowledgments}

This work was carried out in accordance with the requirements of the National Natural Science Foundation of China (61873247) funded project, corresponding test experiments were carried out in the State Key Laboratory of Electronic Testing Technology of North China University, Key 
Laboratory of Instrumental Science and Dynamic Testing of North China University, Huaihai Industry Group in Changzhi City, Shanxi Province, and Alashan Shooting Range. During this period, the authors also got instructions from Professor Zhang Xiaoming and Teacher Li Xiuyuan. The authors would like to thank them for their help and support.

\section{References}

[1] L. An, L. Wang, and D. Zhao, "Attitude determination method of spinning projectile based on geomagnetic azimuth," Journal of Chinese Inertial Technology, vol. 27, no. 5, pp. 618-624, 2019.

[2] A. Grosz, E. Paperno, S. Amrusi, and B. Zadov, "A three-axial search coil magnetometer optimized for small size, low power, and low frequencies," IEEE Sensors Journal, vol. 11, no. 4, pp. 1088-1094, 2011.

[3] H. Liu, H. Dong, J. Ge, B. Bai, Z. Yuan, and Z. Zhao, "Research on a secondary tuning algorithm based on SVD \& STFT for FID signal," Measurment Science and Technology, vol. 27, no. 10, pp. 0957-0233, Article ID 105006, 2016.

[4] J. Shang, D. Zhihong, M. Fu, and S. Wang, "A high-spin rate measurement method for projectiles using a magnetoresistive sensor based on time-frequency domain analysis," Sensors, vol. 16, no. 6, p. 894, 2016.

[5] C. Mateo and J. A. Talavera, "Short-time fourier transform with the window size fixed in the frequency domain," Digital Signal Processing, vol. 77, no. 6, pp. 13-21, 2018.

[6] X. Yan, G. Chen, and X. Tian, "Two-step adaptive augmented unscented Kalman filter for roll angles of spinning missiles based on magnetometer measurements," Measurement and Control, vol. 51, no. 3-4, pp. 73-82, 2018.

[7] T. Addabbo, R. Biondi, S. Cioncolini, A. Fort, F. Rossetti, and V. Vignoli, "A zero-crossing detection system based on FPGA to measure the angular vibrations of rotating shafts," IEEE Transactions on Instrumentation and Measurement, vol. 63, no. 12, pp. 3002-3010, 2014.

[8] Y. Zhou, X. Zhang, and W. Xiao, "Spinning projectile's angular measurement using crest and trough data of a geomagnetic sensor," Measurment Science and Technology, vol. 29, no. 9, Article ID 095007, 2018.

[9] H. Zhao, Z. Su, F. Liu, C. Li, Q. Li, and N. Liu, "Extraction and filter algorithm of roll angular rate for high spinning projectiles," Mathematical Problems in Engineering, vol. 2019, Article ID 3181727, 15 pages, 2019.

[10] S. Carletta and P. Teofilatto, "Design and numerical validation of an algorithm for the detumbling and angular rate determination of a CubeSat using only three-axis magnetometer data," International Journal of Aerospace Engineering, vol. 2018, Article ID 9768475, 12 pages, 2018.

[11] L.-B. Li, M.-X. Li, L.-X. Jiang, D.-Y. Wang, F. Zhan, and T. Sheng, "Angular rate estimation and damping control of satellite with magnetometer data," Optik, vol. 180, no. 11, pp. 1049-1055, 2019.

[12] H. Ma and S. Xu, "Magnetometer-only attitude and angular velocity filtering estimation for attitude changing spacecraft," Acta Astronautica, vol. 102, no. 5, pp. 89-102, 2014.

[13] S. Sabzevari, M. R. Arvan, A. R. Vali, S. M. M. Dehghan, and M. H. Ferdowsi, "Symmetry preserving nonlinear observer for attitude estimation with magnetometer only," ISA Transactions, vol. 102, no. 3, pp. 314-324, 2020.
[14] J. M. Maley, "Efficient attitude estimation for a spin-stabilized projectile," Journal of Guidance Control and Dynamics, vol. 39, no. 2, pp. 1-12, 2016.

[15] G. Hu, W. Wang, Y. Zhong, B. Gao, and C. Gu, "A new direct filtering approach to INS/GNSS integration," Aerospace Science and Technology, vol. 77, no. 7, pp. 755-764, 2018.

[16] M. Yunjian, X. Changfan, J. Yixian, W. Yao, and Z. Yi, "Angular velocity estimation of rolling-ammunition based on magnetometer," Journal of Projectiles, Rockets, Missiles and Guidance, vol. 36, no. 1, pp. 69-72, 2016.

[17] G. Hu, L. Ni, B. Gao, X. Zhu, W. Wang, and Y. Zhong, "Model predictive based unscented Kalman filter for hypersonic vehicle navigation with INS/GNSS integration," IEEE Access, vol. 8, no. 1, pp. 4814-4823, 2016.

[18] C. Chunhang, L. Chunsheng, J. Wendou et al., "The projectile attitude measuring method based on geomagnetic sensor," Journal of Detection \& Control, vol. 40, no. 12, pp. 4814-4823, 2020.

[19] X. Chao, X.-z. Bu, and Y. Bo, “Three different attitude measurements of spinning projectile based on magnetic sensors," Measurement, vol. 47, no. 1, pp. 331-340, 2014.

[20] W. Yu, "Half-experiential formulas for calculating decreasing angular velocity of projectile in trajectory," Journal of Detection and Control, vol. 231, no. 5, pp. 866-876, 2003.

[21] J. Yu, X. Bu, C. Xiang, and B. Yang, "Spinning projectile's attitude measurement using intersection ratio of magnetic sensors," Proceedings of the Institution of Mechanical Engineers Part G-Journal of Aerospace Engineering, vol. 231, no. 5, pp. 1-6, 2016.

[22] X. Zhao, X. Zhang, D. Long, Z. Bai, and Y. Wang, “The design of roll angle magnetic measurement system used in spinning projectiles," Chinese Journal of Sensors and Actuators, vol. 26, no. 9, pp. 1309-1313, 2013. 